\title{
Amphoteric center of luminescence in CdS
}

\author{
O.O. Artem'jeva, O.V. Vakulenko, O.I. Dacenko \\ Taras Shevchenko Kyiv National University, Physics Department \\ 2, prospect Academician Glushkov, 03022 Kyiv, Ukraine, \\ Phone: 38044 5134058,e-mail:doce@phys.univ.kiev.ua
}

\begin{abstract}
The impurity photoluminescence of cadmium sulphide crystals is studied. The luminescence intensity dependences on temperature from 80 to $300 \mathrm{~K}$ are obtained for the bands at 1.7 and $2 \mathrm{eV}$. The thermal curve of $1.7 \mathrm{eV}$ luminescence intensity has a peak between 100 and $150 \mathrm{~K}$. Both these bands are associated with emission of the center based on the $\mathrm{V}_{\mathrm{Cd}}$-type intrinsic point defect. The results can be explained within the framework of the theory of amphoteric centers of charge carrier recombination.
\end{abstract}

Keywords: cadmium sulphide, photoluminescence, amphoteric center of recombination.

Manuscript received 17.03.05; accepted for publication 18.05.05.

\section{Introduction}

As a rule, the intrinsic defects of semiconductor compounds are multicharge centers [1]. Thus, a Zn vacancy in ZnSe can be in three charge states, i.e., " 0 ", " $-e$ ", and " $-2 e$ ", so it is a multicharge donor. It is found that the semiconductors with the multicharged, in particular amphoteric, centers manifest abnormal luminescent properties. In this respect, the crystals $\mathrm{ZnSe}(\mathrm{Te})$ are studied adequately $[2,3]$ when they are used as materials for manufacturing the effective scintillators for the $\mathrm{X}$ - and $\gamma$-rays detectors. Addition of Te into $\mathrm{ZnSe}$ stabilizes the $\mathrm{V}_{\mathrm{Zn}}$ which cause the "working” luminescence band at $640 \mathrm{~nm}$.

The aim of this work is to find similar properties in $\mathrm{CdS}(\mathrm{Te})$ which can also be considered as a scintillation material.

\section{Experiment}

The impurity luminescence of the CdS samples (2.53 eV bandgap at $300 \mathrm{~K}$ [4]) was excited by $488 \mathrm{~nm}$ (2.54 eV) $\mathrm{Ar}^{+}$laser. $33 \AA / \mathrm{mm}$ diffraction spectrometer with the spectral range from 1000 down to $330 \mathrm{~nm}$ (1.2 to $3.7 \mathrm{eV}$ ) was used for the spectral studies. The photoluminescence (PL) spectra were corrected for the spectral sensitivity of the setup.

The temperature dependence of the luminescence intensity was measured from 80 to $300 \mathrm{~K}$ during the natural heating the sample after evaporation of the liquid nitrogen. In so doing, the rate of temperature rise did not exceed $4 \mathrm{~K}$ per minute.

\section{Results}

The PL spectra of the cadmium sulphide samples at room temperature are shown in Fig. 1. One can see that the material doped by the isovalent impurity of tellurium has more intense luminescence. The red band with a peak at 1.6 to $1.8 \mathrm{eV}$ dominates in the spectra of these samples. In addition, the infrared band at $1.3 \mathrm{eV}$ manifests itself in the spectra of the undoped samples where the red luminescence efficiency is essentially less.

The temperature measurements of PL were performed for the $\mathrm{CdS}(\mathrm{Te})$ sample No.14. It was found that the red luminescence yield rises as the temperature decreases, furthermore, at $2.0 \mathrm{eV}$, a yellow band appears in the spectrum and dominates at low temperatures (Fig. 2).

The studies have shown that the yellow luminescence intensity increases monotonously when the temperature decreases from 300 down to $80 \mathrm{~K}$ (Fig. 3). At the same time, the temperature dependence of the low-energy luminescence band at $1.6 \mathrm{eV}$ is anomalous and has a peak.

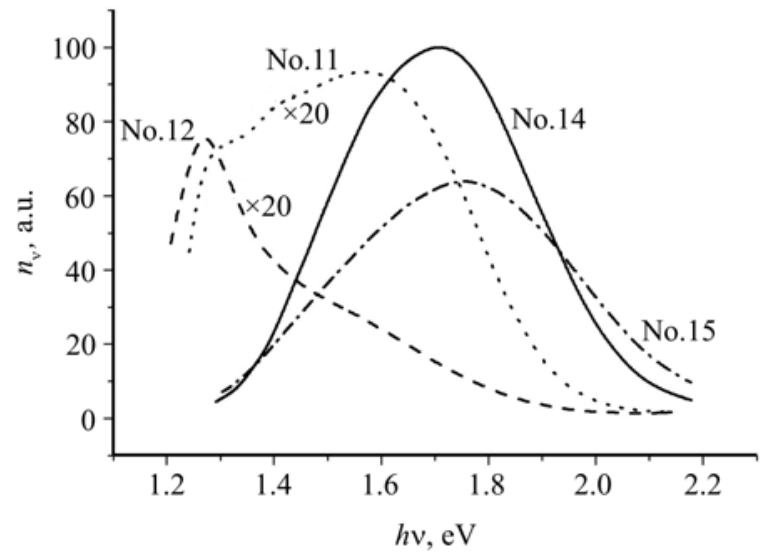

Fig. 1. PL spectra of the CdS (No.11 and 12) and CdS(Te) (No.14 and 15) samples at the room temperature. 


\section{Discussion}

The red PL efficiency in the samples with the Te isovalent impurity is essentially higher than that in the undoped material. The chalcogene impurity addition into II-VI compounds is known [1] to stabilize the metal vacancies and the complexes related to them. Therefore, we suggest that, in our material, the red luminescence center is also the metal vacancy, i.e. $\mathrm{V}_{\mathrm{Cd}}$, or some complex including this intrinsic point defect.

The temperature measurement results suggest that the above luminescence center is amphoteric [5] and it can be at three charge states, namely, “+“, "0”, and “-”. It can correspondingly be both the donor and the acceptor and change its impurity type together with the charge. In $\mathrm{ZnSe}(\mathrm{Te})$, the $\left\langle\mathrm{V}_{\mathrm{Zn}}^{-} \mathrm{Te}_{\mathrm{Se}}^{0} \mathrm{D}^{+}\right\rangle^{0}$ associate is such a center. Both an unintential impurity and intrinsic atom of the lattice located in the interstice can play the role of the donor, e.g., $\mathrm{Zn}_{i}{ }^{+}$in $\mathrm{ZnSe}$ or $\mathrm{Cd}_{i}{ }^{+}$in CdS. As it was mentioned above, the single vacancy of the chalcogen has the donor properties, too.

The luminescence occurs in the crystal according to the scheme is presented in Fig. 4 [6]. An amphoteric center has two energy levels in the semiconductor bandgap, that is the donor one corresponding to the charge states "+" and " 0 " and the acceptor one ("-" and "0"). It should be noted that the acceptor level is located above the donor one. The positively charged center can trap an electron from the conduction band (arrow 2), which makes it neutral. We suggest that this thansition causes the yellow luminescence band close to $2.1 \mathrm{eV}$. The center can change the positive charge state to the neutral one also due to the thermal trapping a valence band electron (arrow 3). This transition decreases the number of positively charged centers $N^{+}$and, hence, reduces the intensity of the yellow luminescence band which is proportional to $N^{+}$. Therefore, this band is observed at low temperatures (see Fig. 2) when the probability of the transition is low.

The neutral center can donate an electron to the valence band (arrow 4) and resume a positive charge but it can trap one more free electron (arrow 5) and acquire a negative charge. In doing so, the electron transfers to the higher, i.e., acceptor level and the quantum of the lower energy (about $1.7 \mathrm{eV}$ ) is emitted, which causes the red luminescence of cadmium sulphide. The anomalous temperature dependence of its intensity at low temperatures (see Fig. 3) can be explained by probability decrease of the transition 3 (Fig. 4). This transition increases the number of neutral centers $N^{0}$ and the red band intensity is proportional to this value. When the probabilities of neutralization of the negatively charged center (transition 6) are small, the band intensity must reduce while the temperature decreases.

It should be noted that the theoretical studies confirm the possibility of that kind of anomaly. In particular, they have been performed to theoretically justify the $640 \mathrm{~nm}$ luminescence center in ZnSe being multicharged [3].

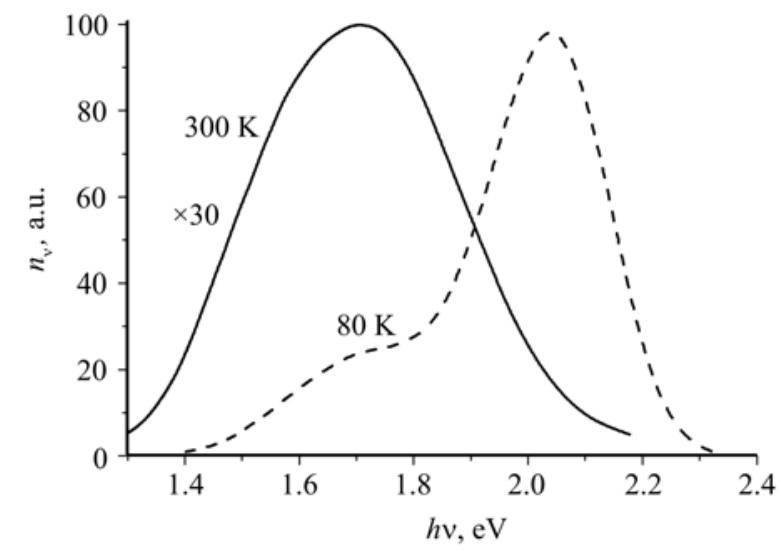

Fig. 2. PL spectra of the CdS(Te) sample No.14 at the room and liquid nitrogen temperatures.

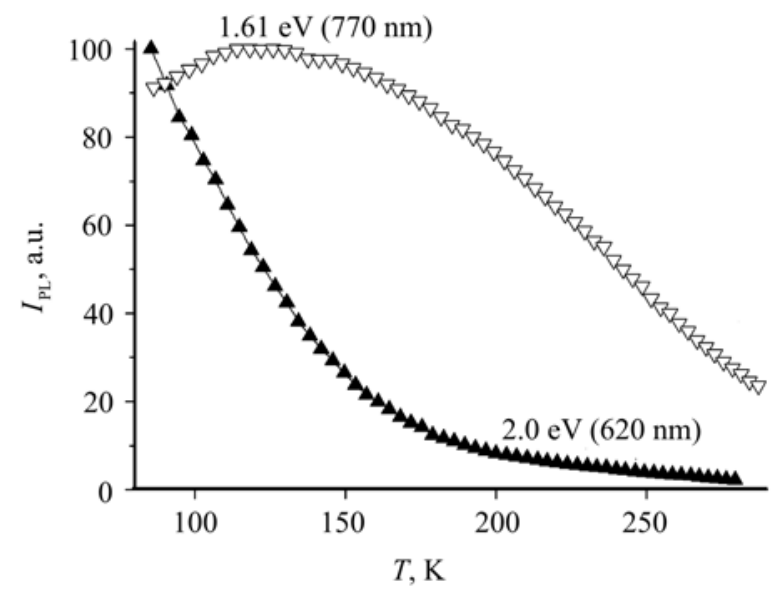

Fig. 3. The temperature dependences of PL intensity of CdS(Te) sample No.14 in different parts of the spectrum.

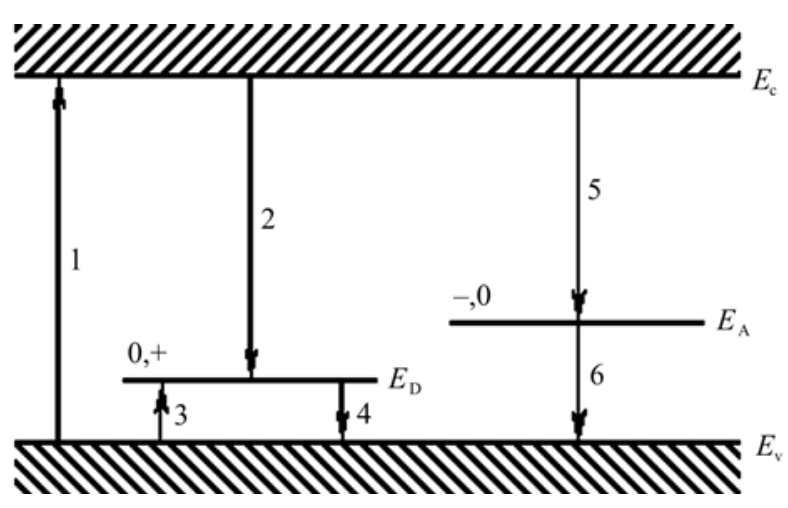

Fig. 4. Diagram of the electron transitions in the semiconductor with the amphoteric impurity [6]: excitation (1), radiative transition of an electron to the donor level of the positively charged center (2), exchange processes between the donor level and the valence band $(3,4)$, radiative electron transition to the acceptor level of the neutral luminescence center (5) and neutralization of the negatively charged center (6). 


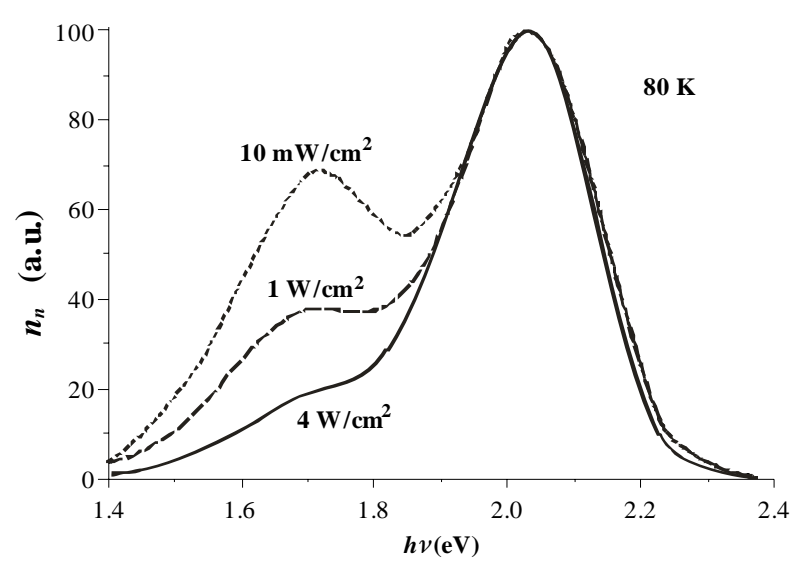

Fig. 5. Low-temperature PL spectra of the CdS(Te) sample No.14 at different excitation intensities. The spectra are normalized by the yellow band intensity.

The differences in the luminescence spectra of the sample at different excitation powers (Fig. 5) can be also explained within the suggestion that there is the amphoteric luminescence center in CdS. The number of luminescence transitions 2 and 5 (see Fig. 4) in reference to the impurity levels increases while the excitation intensity rises, and the luminescence centers are saturated with electrons. Although the luminescence intensity increases further when the excitation builds up, this increase is sublinear, i.e., the quantum yield of both bands reduces. Since the probability of the acceptor level neutralization (transition 6) ) is low (essentially less than the probability of emptying the donor level, transition 4), according to our supposition, the longwave PL yield reduces more rapidly than the short-wave one. This fact is shown in Fig. 5, the red band intensity decreases in comparison with the yellow one as the excitation intensity increases.

\section{Conclusions}

The dependence of $2 \mathrm{eV}$ yellow luminescence band intensity on the temperature is normal in the range from 80 to $300 \mathrm{~K}$, whereas the thermal curve of $1.7 \mathrm{eV}$ red band intensity manifests the anomaly as a peak. The relative intensity of the red luminescence band reduces when the excitation intensity rises. The red and yellow luminescence bands in CdS are caused by amphoteric center. The cadmium vacancy or a complex based on it can be such a center.

\section{References}

1. V. Ryzhykov, High-efficiency semiconductor scintillation detectors based on II-VI compounds, NIITEKhIM, Moscow (1984) (in Russian).

2. O.V. Vakulenko, V.D. Ryzhykov, B.M. Shutov, Nature of the long-wave luminescence of $\mathrm{ZnSe}<\mathrm{Te}>$ :Zn // Belorus. J. Appl. Spectrosc. 49 (3), p. 439-444 (1988).

3. O.V. Vakulenko and V.M. Kravchenko, Luminescent properties of semiconductors with amphoteric centers of recombination // Phys. status solidi $(b)$ 211, p. 839-846 (1999).

4. V.V. Serdjuk, Ju.F. Vaksman, Semiconductor luminescence, Vyshcha Shkola, Kiev-Odessa (1988) (in Russian).

5. O.V. Vakulenko, M.P. Lysytsa, Optical recharging the impurity in semiconductors, Naukova Dumka, Kyiv, (1992) (in Russian).

6. O.V. Vakulenko, V.N. Suprunenko, V.D. Ryzhykov, Kinetics of the impurity luminescence in semiconductors with amphoteric centers of recombination // Ukr. J. Phys. 35 (10), p.14851489 (1990) 